\title{
D- $\alpha$-Tocopheryl polyethylene glycol succinate/ Solutol HS I5 mixed micelles for the delivery of baohuoside I against non-small-cell lung cancer: optimization and in vitro, in vivo evaluation
}

International Journal of Nanomedicine

8 September 2016

Number of times this article has been viewed

Hongmei Yan ${ }^{1,2}$

Zhenhai Zhang ${ }^{2}$

Xiaobin Jia ${ }^{1,2}$

Jie Song ${ }^{1,2}$

'College of Pharmacy, Nanjing University of Chinese Medicine, ${ }^{2}$ Key Laboratory of New Drug Delivery System of Chinese Materia Medica, Third School of Clinical Medical of Nanjing University of Chinese Medicine, Nanjing, People's Republic of China
Correspondence: Xiaobin Jia; Jie Song Key Laboratory of New Drug Delivery System of Chinese Materia Medica, Third School of Clinical Medical of Nanjing University of Chinese Medicine, 100 Shizi Road, Nanjing, Jiangsu 210028, People's Republic of China

Tel +86258560 8672; +86258327 II 02

Fax +86258563 7809; +862585309785

Email jiaxiaobinpharmacy@।63.com;

momo198420@hotmail.com

\begin{abstract}
Baohuoside I, extracted from the Herba epimedii, is an effective but a poorly soluble antitumor drug. To improve its solubility, formulation of baohuoside I-loaded mixed micelles with D- $\alpha$-tocopheryl polyethylene glycol succinate and Solutol HS 15 (BTSM) has been developed in this study. We performed a systematic comparative evaluation of the antiproliferative effect, cellular uptake, antitumor efficacy, and in vivo tumor targeting of these micelles using non-small-cell lung cancer (NSCLC) A549 cells. Results showed that the obtained micelles have a mean particle size of $\sim 62.54 \mathrm{~nm}$, and the size of micelles was narrowly distributed. With the improved cellular uptake, BTSM displayed a more potent antiproliferative action on A549 cell lines than baohuoside I; half-maximal inhibitory concentration was 7.83 vs $20.37 \mu \mathrm{g} / \mathrm{mL}$, respectively. The antitumor efficacy test in nude mice showed that BTSM exhibited significantly higher antitumor activity against NSCLC with lesser toxic effects on normal tissues. The imaging study for in vivo targeting demonstrated that the mixed micelles formulation achieved effective and targeted drug delivery. Therefore, BTSM might be a potential antitumor formulation.
\end{abstract}

Keywords: baohuoside I-loaded mixed micelles, TPGS, Solutol HS 15, antitumor

\section{Introduction}

Herba epimedii has been traditionally used in the People's Republic of China as a tonic, an aphrodisiac, and an antirheumatic drug for many years. Baohuoside I (also known as icariside II) is the main active flavonoid component of Herba epimedii. ${ }^{1-3}$ Baohuoside I can induce apoptosis in human non-small-cell lung cancer (NSCLC) cells via reactive oxygen species-mediated mitochondrial pathway and also inhibits the growth of U266 multiple myeloma and preosteoclastic RAW264.7 cells. ${ }^{4-7}$

Baohuoside I has very poor solubility in water. Moreover, baohuoside I possesses a low absorptive permeability and a high rate of efflux via apical efflux transporters such as multidrug resistance-associated proteins 1 and 2 (MRP 1 and MRP 2) and P-glycoprotein (P-gp). However, the membrane permeability of baohuoside I is slightly better than that of the other flavonoids in Herba epimedii, and the rate of efflux $\left(P_{\mathrm{BA}} / P_{\mathrm{AB}}\right)$ of baohuoside I reached 9.84 in a previous study using Caco- 2 cells. ${ }^{8}$ These peculiarities restrict the application of baohuoside I in the management of cancer therapy. Thus, poor aqueous solubility, low membrane permeability, and a severe efflux phenomenon all limit the therapeutic use of baohuoside I in humans., ${ }^{9,10}$ 
Therefore, improving the aqueous solubility and membrane permeability of baohuoside I and reducing its efflux phenomenon are essential for determining the future applications of baohuoside I. In our previous studies, various drug delivery systems, including nanoparticles and $\mathrm{D}-\alpha-$ tocopheryl polyethylene glycol succinate (TPGS) complexes, have been developed to solve the aforementioned limitations of baohuoside I. ${ }^{69}$ Among the several micellar formulations evaluated as carriers of anticancer drugs, mixed micelles are the appropriate choice for the carriers of baohuoside I. Micellar systems have many advantages such as increasing the drug solubility, circumventing the uptake by the reticuloendothelial system, improving circulation time, and passive tumor targeting by the enhanced permeability and retention (EPR) effect. ${ }^{11,12}$ In addition, mixed micelles have synergistic properties such as increased drug stability and drug loading efficiency (DE) compared to that of the individual components. ${ }^{13,14}$ To the best of our knowledge, no studies have focused on the influence of mixed micelles for the delivery of baohuoside I. Therefore, we prepared mixed micelles of baohuoside I consisting of TPGS and a novel drug carrier material Solutol HS 15, to increase the aqueous solubility and membrane permeability and to improve the efficacy of baohuoside I.

Vitamin E TPGS or simply TPGS is a water-soluble derivative of natural vitamin $\mathrm{E}$, which is formed by esterification of vitamin E succinate with polyethylene glycol (PEG). In the recent years, TPGS has been extensively used for developing various drug delivery systems, extending the half-life of drugs in the plasma, and increasing the cellular uptake of drugs. ${ }^{15,16}$ Therefore, TPGS can be used as an ideal molecular biomaterial for developing various drug delivery systems, including prodrugs, micelles, liposomes, and nanoparticles, which would enable sustained, controlled, and targeted drug delivery. Moreover, TPGS has been used as an excipient for overcoming multidrug resistance (MDR) and as an inhibitor of P-gp. ${ }^{17}$

A nonionic surfactant called Solutol HS 15 (polyoxyethylene esters of 12-hydroxystearic acid) is structurally made by fusing fatty acids and end-capped methoxy polyethylene glycol (mPEG), which are connected via chemically and biologically labile linkers. Solutol HS 15 is a new type of amphiphilic surfactant showing high performance, low toxicity, and excellent biocompatibility. ${ }^{18}$ The solubility capacity against hydrophobic drug showed a linear growth as the solvent concentration is increased. Moreover, no matter what the solubility or chemical structure of drug is, the particles of micelles, finally formed, will remain unchanged. Therefore, the high solubility capacity of Solutol HS 15 enables the injection of high-dose drug in low volume. In addition, there is no need to take an antihistamine and corticosteroids before its use. The low hemolytic property of Solutol HS 15 suggests that it is associated with low toxicity and irritation. ${ }^{19}$ Solutol HS 15 has been reported to modulate the cytotoxicity and the accumulation of anticancer drugs by P-gp inhibition. ${ }^{20}$ PEG seems to be a selective and potent modulator of organic anion transporting polypeptides $1 \mathrm{~A} 2$ with half-maximal inhibitory concentration $\left(\mathrm{IC}_{50}\right)$ values of $0.05 \%$ (taurocholate) and $0.14 \%$ (estrone-3-sulfate). It has been shown to interact with cytochrome P450-dependent drug metabolism as well as efflux transporters such as P-glycoprotein (ABCB1) and MDR-associated protein $2(\mathrm{ABCC} 2) .{ }^{21} \mathrm{It}$ is suspected that this inhibition is mediated by changing the plasma membrane fluidity of MDR cells.

To increase the solubility and membrane permeability and enhance the anticancer effects of baohuoside I, we prepared baohuoside I-loaded mixed micelles consisting of TPGS and Solutol HS 15 (BTSM) by using a solvent evaporation method and optimized the preparation process. We selected the NSCLC A549 cells resided in the right oxter to investigate the in vitro and in vivo anticancer effects of baohuoside I-loaded mixed micelles when compared to baohuoside I. In addition, we examined in vivo targeting behavior of the mixed micelles in this study.

\section{Materials and methods Materials}

Baohuoside I was obtained from the Nanjing Zelang Medical Technology Co. Ltd. (Nanjing, People's Republic of China) and purity was $98.22 \%$. TPGS was purchased from Sigma-Aldrich Co. (St Louis, MO, USA). Solutol HS 15 was purchased from BASF AG (ludwigshafen, Germany). Plastic cell culture dishes and plates were purchased from Corning Incorporated (Corning, NY, USA). Coumarin-6 and 4,6-diamidino-2-phenylindole (DAPI) were purchased from Sigma-Aldrich Co. 1,1-Dioctadecyl-3,3,3, 3-tetramethylindotricarbocyanine (DiR) was purchased from Ganhua Trade Co. Ltd. (Shanghai, People's Republic of China). All reagents were of analytical grade except methanol, which was of chromatographical grade.

\section{HPLC analysis}

The concentration of baohuoside I in the dissolution medium was determined by high-pressure liquid chromatography (HPLC, Agilent 1260) equipped with a Hedera RP-C18 column $(250 \times 4.6 \mathrm{~mm}, 5 \mu \mathrm{m})$. The mobile phase of methanol and water $(75: 25, \mathrm{v}: \mathrm{v})$ was used at a flow rate of $1.0 \mathrm{~mL} \cdot \mathrm{min}^{-1}$. The ultraviolet (UV) detector was set at $270 \mathrm{~nm}$ to analyze the column effluent and the column temperature was $30^{\circ} \mathrm{C}$. The entire solution was filtered through a $0.45 \mu \mathrm{m}$ membrane filter (EMD Millipore, Billerica, MA, USA) and degassed 
prior to use. The injection volume was $10 \mu \mathrm{L}$. The recovery rates for baohuoside I were in the range of 99\%-101\%, and the relative standard deviation was less than $2 \%$. Intra- and interday precisions for baohuoside I were below $2 \%$.

\section{Preparation of baohuoside I-loaded mixed micelles}

Baohuoside I-loaded mixed micelles were prepared by a thin-film hydration method. In brief, $50 \mathrm{mg}$ of baohuoside I and $450 \mathrm{mg}$ of a mixture of TPGS and Solutol HS 15 at different ratios $(1: 1,1: 2,1: 4 ; \mathrm{w} / \mathrm{w})$ were dissolved in absolute ethyl alcohol by ultrasonic method in a round-bottom flask. The solvent was subsequently evaporated by rotary evaporation to obtain a thin film. The film was then kept in a vacuum overnight at room temperature to remove the residual ethanol. The dried film was hydrated with $20 \mathrm{~mL} 0.9 \% \mathrm{NaCl}$ solution and a clear micelle solution was obtained. The solution was then filtrated through $0.22 \mu \mathrm{m}$ membrane filter to remove the nonincorporated baohuoside I and was followed by lyophilization. The mixed micelles prepared by this method disperse well in water.

\section{Baohuoside I loading efficiency and encapsulation ratio}

Briefly, $20 \mu \mathrm{L}$ of baohuoside I-loaded micelles were mixed with $80 \mu \mathrm{L}$ of methanol for micelles disruption and dissolved in mobile phase consisting of acetonitrile and deionized water $(50: 50, \mathrm{v} / \mathrm{v})$ to $1 \mathrm{~mL}$. The solution was filtered through $0.45 \mu \mathrm{m}$ syringe filter before being transferred to HPLC vial. All samples were analyzed in triplicate. The encapsulation efficiency (EE) and the DE were calculated using the following equations:

$\begin{gathered}\text { Encapsulation } \\ \text { efficiency (\%) }\end{gathered}=\frac{\begin{array}{c}\text { Weight of baohuoside I } \\ \text { in micelles }\end{array}}{\begin{array}{c}\text { Weight of baohuoside I } \\ \text { fed initially }\end{array}} \times 100 \%$

$$
\begin{gathered}
\text { Drug loading } \\
\text { efficiency (\%) }
\end{gathered}=\frac{\begin{array}{c}
\text { Weight of baohuoside I } \\
\text { in micelles }
\end{array}}{\text { Total weight of micelles }} \times 100 \%
$$

\section{Characterization of baohuoside I-loaded mixed micelles}

The particle size of all micelles was measured by dynamic light scattering (DLS) using a Zetasizer Nano ZS (Malvern Zetasizer 3000; Malvern Instruments, Malvern, UK). The particle size was carried out in triplicate. The morphological evaluation was performed using transmission electron microscopy (TEM,
H7650; Hitachi Ltd., Tokyo, Japan). The micellar solution was diluted at a concentration of $100 \mu \mathrm{g} / \mathrm{mL}$ and stirred for 24 hours. Samples of baohuoside I-loaded mixed micelles were prepared by taking one drop to film the copper net formation of droplets and dried in the natural air. It was negatively stained with phosphomolybdic acid and observed under the TEM.

\section{In vitro baohuoside I release}

The in vitro release behavior of baohuoside I from baohuoside I-loaded mixed micelles was monitored in a phosphate-buffered saline (PBS, $\mathrm{pH}$ 7.4) medium containing $0.5 \%$ Tween 80 to obtain pseudo-sink conditions. Briefly, aliquots of baohuoside I mixed micelles were introduced into a dialysis bag with a molecular weight cutoff of 3,500 Da (Green bird Inc., Shanghai, People's Republic of China), the sealed end of which was immersed fully into $50 \mathrm{~mL}$ of a release medium at $37^{\circ} \mathrm{C}$; this medium was stirred at $100 \mathrm{rpm}$ for 24 hours. At fixed time intervals, $0.5 \mathrm{~mL}$ aliquots were withdrawn and replaced with an equal volume of fresh medium. Baohuoside I release from the stock solution was performed under the same conditions as the controls. The concentration of baohuoside I in the samples was determined by HPLC as described earlier, with correction for the volume replacement.

\section{Cell lines and cell culture}

The human lung adenocarcinoma cell line A549 was obtained from Nanjing KeyGen Biotech. Co. Ltd. (Nanjing, People's Republic of China) which was cultured in Dulbecco's Modified Eagle's Medium (DMEM) containing $10 \%$ fetal bovine serum, $100 \mathrm{IU} / \mathrm{mL}$ penicillin, and $100 \mu \mathrm{g} / \mathrm{mL}$ streptomycin sulfates at $37^{\circ} \mathrm{C}$ in $5 \% \mathrm{CO}_{2}$.

\section{Animals and tumor implantation}

This study and the experimental protocols were approved by the Institutional Animal Care and Use Committee (IACUC) of Jiangsu Provincial Academy of Chinese Medicine under the approval number JPA-141012-5. Male BALB/c nude mice $(22 \pm 2 \mathrm{~g})$ were obtained from Changzhou Cavens Lab Animal Co. Ltd. (Changzhou, People's Republic of China). All animal-handling procedures were performed in accordance with the guidelines of the 'Law for The Care and Welfare of Animals in China' and approved by the Institutional Animal Care and Use Committee (IACUC) of Jiangsu Provincial Academy of Chinese Medicine under the approval number JPA-141012-5. Pathogen-free BALB/c mice were housed in separate cages with normal access to food and water and kept on a 12-hour light-dark cycle. To generate tumors, the flanks of 6-week-old male BALB/c mice were shaved and $200 \mu \mathrm{L}$ of single cell suspension containing $1 \times 10^{6}$ A549 
cells in serum-free DMEM was injected subcutaneously under anesthesia. ${ }^{22}$

\section{In vitro cytotoxicity assay}

The cytotoxicity of baohuoside I and BTSM against A549 cells was measured using the MTT assay. The cells $(100 \mu \mathrm{L}$ of single cell suspension containing $1 \times 10^{5} \mathrm{~A} 549$ cells) were seeded into a 96 -well plate. After incubation for 24 hours, the cells were treated with baohuoside I at a range of concentrations $(0,2,6,8,12,16,24,32,48$, and $64 \mu \mathrm{g} / \mathrm{mL})$ for 24,48 , and 72 hours. Then, $10 \mu \mathrm{L}$ MTT $(5 \mathrm{mg} / \mathrm{mL})$ was added to each well. After incubation for 4 hours, the medium was removed and replaced with $100 \mu \mathrm{L}$ dimethyl sulfoxide solution to dissolve. The absorbance of each well was measured by a SpectraMax 190 microplate reader (Molecular Devices LLC, Sunnyvale, CA, USA) at the wavelengths of 570 and $630 \mathrm{~nm}$. Each experiment was repeated three times.

\section{In vitro cellular uptake HPLC analysis}

The A549 cells were seeded into a six-well culture plate. After incubation for 24 hours, the original culture medium was removed, and the cells were treated with baohuoside I formulations at a concentration of $25 \mu \mathrm{M}$ and incubated for 1,2 , and 4 hours to evaluate the effects of the relationship between uptake and uptake time. After incubation, the medium was discarded, and the cells were extensively washed three times in cold PBS to remove noningested drugs. Then, $1 \mathrm{~mL}$ of distilled water was added and cells were scraped. The suspended fluid of cells was collected and placed in an ice bath, subjected to ultrasonic cell disruption, centrifuged, and the supernatant was taken in another clean centrifuge tube. The supernatant was dried with nitrogen, dissolved in methanol, and filtered with $0.45 \mu \mathrm{m}$ Millipore filter; the volume was made up to $1 \mathrm{~mL}$, and HPLC was used to measure the content of baohuoside I.

\section{Fluorescence study}

Coumarin-6-M (coumarin-6-loaded mixed micelles composed of TPGS and Solutol HS 15) was prepared by a thin-film hydration method according to the prescription of baohuoside I-loaded mixed micelles, the ratio of coumarin6-M: TPGS: Solutol HS 15 was 1:1.8:7.2 (w/w). In vitro qualitative study with regard to the cellular uptake of the micellar formulations was assessed by the fluorescence microscope. After reaching confluence, cells were detached, counted, and seeded in a 24-well plate overnight. Then, the medium was replaced with coumarin-6-loaded micelles suspension at a concentration of $0.2 \mathrm{mg} / \mathrm{mL}$ and incubated for 2 hours. The cells were washed twice with prewarmed PBS and fixed with $95 \%$ ethanol for 20 minutes. Then, the cells were washed twice with PBS and the nuclei were counterstained with DAPI for 30 minutes. The cells were washed again twice with PBS and immersed in PBS for microscopic imaging.

\section{In vivo antitumor activity evaluation}

For in vivo implantation, nude mice were subcutaneously injected in the right flank with $0.2 \mathrm{~mL}$ of cell suspension containing $1.7 \times 10^{6} \mathrm{~A} 549$ cells. The in vivo antitumor studies were started when the tumor volumes reached $\sim 60 \mathrm{~mm}^{3}$ (designated as day 0$)$. Mice were randomly divided into three groups $(n=6)$ : group 1 for saline solution, group 2 for baohuoside I $(10 \mathrm{mg} / \mathrm{kg})$, and group 3 for BTSM $(10 \mathrm{mg} / \mathrm{kg}$ ). Mice were administered according to their group protocol through the tail vein for five times every 2 days. Tumor volume and mouse weight were monitored daily. Tumor volume was calculated by the equation $\mathrm{V}=\left(\mathrm{a} \times \mathrm{b}^{2}\right) / 2$, where a represents the longest diameter and $\mathrm{b}$ represents the shortest diameter perpendicular to length. At the end of the experiment, the animals were sacrificed and the tumor, thymus, and spleen masses were harvested and weighed.

\section{In vivo imaging}

DiR-M (DiR-loaded mixed micelles composed of TPGS and Solutol HS 15) was prepared by a solvent evaporation method according to the prescription of baohuoside I-loaded mixed micelles. When tumors reached an acceptable size, DiR-M was injected into the tail vein of the tumor-bearing mice at a dose of $5.0 \mathrm{mg} / \mathrm{kg}$ to investigate the in vivo distribution. The mice were anesthetized and imaged at predetermined time points $(2,4,8,12$, and 24 hours) after intravenous injection using the Maestro in vivo imaging system (NightOWL II LB983; Berthold Technologies, Bad Wildbad, Germany).

\section{Statistical analysis}

Data were expressed as mean \pm standard deviation. Statistical analysis was performed by Kruskal-Wallis test. $P$-value $<0.05$ was considered statistically significant.

\section{Results}

\section{Formulation and characterization of mixed micelles}

We prepared baohuoside I-loaded mixed micelles by using a thin-film hydration method (Figure 1A). Results showed that the particle size, EE\% and DE\% of BTSM increased with an increase in TPGS proportion. Thus, in consideration of the particle size, $\mathrm{EE} \%$, and $\mathrm{DE} \%$, the ratio of baohuoside 

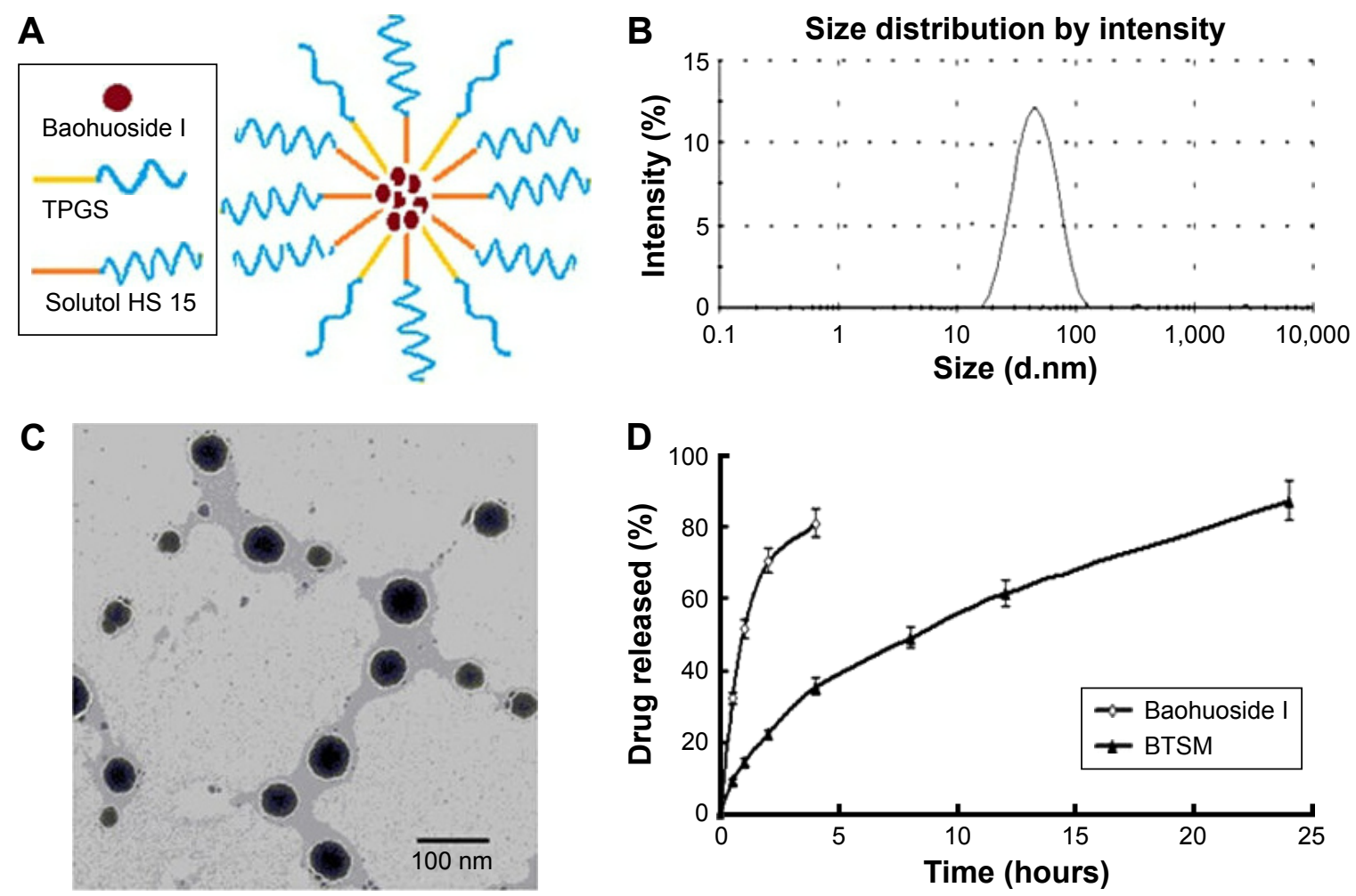

Figure I Characteristics of the BTSM.

Notes: (A) Structure of the baohuoside I mixed micelles. (B) Size distribution of BTSM as determined by dynamic light scattering. (C) Transmission electron microscope (TEM) image of BTSM (scale bar $=100 \mathrm{~nm}$ ). (D) Baohuoside I release profiles from the micelles in vitro in PBS ( $\mathrm{PH}$ 7.4). Data are presented as the mean \pm SD ( $\mathrm{n}=3$ ).

Abbreviations: BTSM, baohuoside I-loaded mixed micelles consisting of TPGS and Solutol HS I5; TPGS, D- $\alpha$-tocopheryl polyethylene glycol succinate; PBS, phosphatebuffered saline; SD, standard deviation.

I:TPGS:Solutol HS 15 used in this study was 1:1.8:7.2 (w/w). BTSM thus prepared had smaller particle size $(\sim 60.52 \mathrm{~nm})$ and higher encapsulation efficiencies (above 85\%) under such conditions (Table1). The mean diameter of BTSM was determined using DLS and TEM (Figure 1B and C, respectively).

The accumulated percentage release of baohuoside I from the active pharmaceutical ingredient baohuoside I and BTSM in PBS (pH 7.4) containing $0.1 \%$ (w/v) Tween 80 is shown in Figure 1D. More than $80 \%$ of baohuoside I in the stock solution was released within the first 4 hours, suggesting that baohuoside I could freely diffuse through the dialysis membrane. BTSM showed no burst release but exhibited sustained drug release over a period greater than 24 hours,

Table I Physicochemical characterizations of baohuoside I-loaded micelles $(n=3)$

\begin{tabular}{llllll}
\hline Batches & Composition & Ratio (w/w) & Size (nm) & EE (\%) & DE (\%) \\
\hline I & Baohuoside & I:4.5:4.5 & $31.52 \pm 1.31$ & 64.32 & 5.31 \\
2 & I:TPGS:Solutol & I:3:6 & $52.47 \pm 2.04$ & 72.75 & 6.83 \\
3 & HS 15 & I:I.8:7.2 & $60.52 \pm 2.25$ & 87.38 & 8.13 \\
\hline
\end{tabular}

Note: aData presented as mean \pm standard deviation $(n=3)$.

Abbreviations: EE, encapsulation efficiency; DE, drug loading efficiency; TPGS, $D$ - $\alpha$-tocopheryl polyethylene glycol succinate. with only $35.82 \%$ of the baohuoside I released in the first 4 hours. After 24 hours of dialysis in PBS (pH 7.4), the percentage of baohuoside I released from the mixed micelles was $87.68 \%$. Compared to baohuoside I, BTSM showed a slow drug release.

\section{In vitro evaluation of baohuoside I micelle formulations}

We examined the in vitro cytotoxicity of baohuoside I formulated in baohuoside I and BTSM and compared it with that of paclitaxel at an equivalent concentration on A549 cells after incubation at $37^{\circ} \mathrm{C}$ for 24,48 , and 72 hours (Table 2). BTSM had lower $\mathrm{IC}_{50}$ values than baohuoside I, which indicated that compared to the free drug, the mixed micelle formulation could significantly increase the cytotoxicity of baohuoside I against A549 cells in vitro. In addition, mixed micelles without the drug did not show any antiproliferative effects when the concentration of micellar materials was higher than $500 \mu \mathrm{g} / \mathrm{mL}$. This indicated that TPGS and Solutol HS 15 showed very low toxicity and irritation.

The quantitative uptake by A549 cells after incubation with baohuoside I-loaded mixed micelles for 1, 2, and 4 hours 
Table $2 \mathrm{IC}_{50}$ values $(\mu \mathrm{g} / \mathrm{mL})$ of free baohuoside I or BTSM against A549 cells $(n=6)$

\begin{tabular}{llll}
\hline $\begin{array}{llll}\text { Incubation } \\
\text { time (hours) }\end{array}$ & $\mathrm{IC}_{50}(\mu \mathrm{g} / \mathrm{mL})$ & & \\
\cline { 2 - 4 } & Paclitaxel & Baohuoside I & BTSM \\
\hline 24 & $4.76 \pm 0.37$ & $20.37 \pm 1.36$ & $7.83 \pm 0.42$ \\
48 & $3.7 \mathrm{I} \pm 0.19$ & $15.84 \pm 0.97$ & $5.58 \pm 0.26$ \\
72 & $2.12 \pm 0.12$ & $11.69 \pm 0.4 \mathrm{I}$ & $4.3 \mathrm{I} \pm 0.22$ \\
\hline
\end{tabular}

Note: Data presented as mean \pm standard deviation $(n=6)$.

Abbreviations: $\mathrm{IC}_{50}$, half-maximal inhibitory concentration; BTSM; baohuoside Iloaded mixed micelles consisting of TPGS and Solutol HS I5; TPGS, D- $\alpha$-tocopheryl polyethylene glycol succinate.

is shown in Figure 2A. We observed a trend toward increased uptake of mixed micelles by the cells with an increase in the incubation time. The cellular uptake of BTSM was significantly higher than the free drug. The qualitative uptake by A549 cells after incubation with coumarin-6-M for 2 hours is shown in Figure 2B. Our results showed that the green fluorescence in the A549 cells, which corresponds to coumarin-6-M, was stronger than that produced by free coumarin- 6 .

\section{In vivo antitumor efficacy}

The micelles showed remarkable antitumor effects in vitro; therefore, we examined the antitumor effects of the micelles after systemic application. The tumor volume-time curve and weight analysis are shown in Figure 3A and C, respectively. Our results showed that tumor growth in the control group was greater than in the baohuoside I groups, which indicated that baohuoside I was efficient in inhibiting tumor growth to varying degrees. Because of the effect of Solutol HS 15, BTSM produced stronger effects than baohuoside I, which could be concluded from the volume of tumors and weights of the mice. The variations in the body weights of the mice were plotted against time as shown in Figure 3B and D. The body weights of mice in the control group and baohuoside I groups slightly increased, which could be related to the increasing tumor weight. However, the amount of increase in weight was less in baohuoside I-treated groups when compared to that in the control group, which might be related to the impact of baohuoside I. The body weights of the mice treated with BTSM decreased, which might be because of the efficient inhibition of tumor growth by BTSM when compared to baohuoside I. In addition, the body weights of the mice in the paclitaxel group decreased to a greater extent compared to those in the BTSM-treated groups, which indicated that BTSM exhibited lesser toxicity to normal tissues than paclitaxel. The results of organ index (percentage of organ weight to body weight of each mouse) in different groups are shown in Figure 3D. Compared to the control group, the baohuoside I group showed a decrease in the organ index of thymus and spleen to varying degrees, which may be attributed to the inherent toxicity of baohuoside I, but the values remained within the tolerance range. BTSM-treated groups showed an increase in the organ index of thymus and spleen when compared to baohuoside I-treated group, which might be due to the reason that micellar systems could circumvent the uptake by reticuloendothelial system and show passive tumor targeting by EPR effect. However, compared to the control group, the paclitaxel group showed a significant decrease in the organ index. Thus, our results showed that compared to paclitaxel, BTSM exhibited lesser toxic effects on normal tissues.

\section{Imaging to determine in vivo targeting of baohuoside I mixed micelles}

To investigate whether mixed micelles composed of TPGS and Solutol HS 15 can specifically target A549 cells in vivo, we established a tumor model by intravenously injecting mice
A

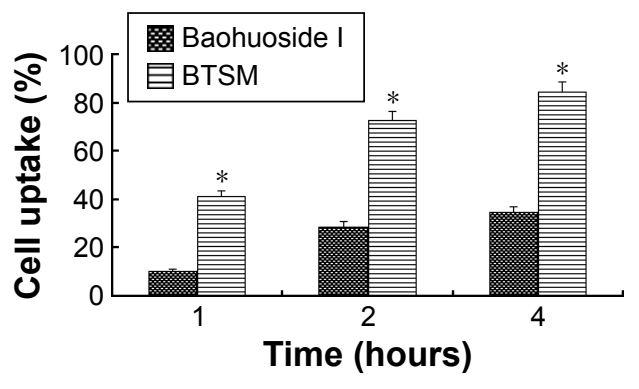

B

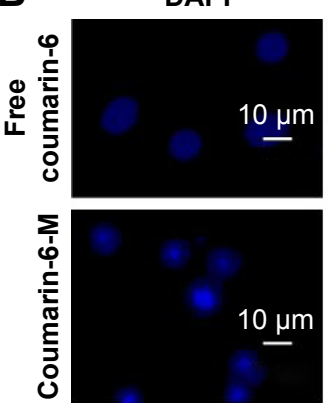

Coumarin-6

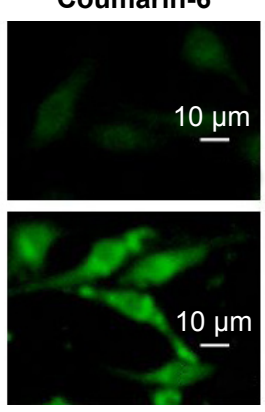

Merge

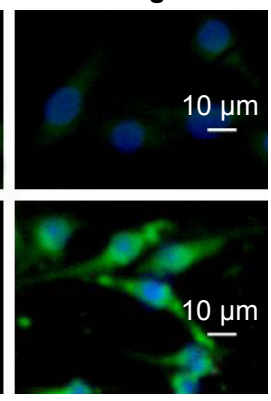

Figure 2 DAPI channels showing the blue fluorescence from propidium iodide stained nuclei, Coumarin-6 channels showing the green fluorescence from the mixed micelles distributed in cytoplasm, and merged channels of Coumarin-6 and DAPI.

Notes: (A) Cellular uptake efficiency of the baohuoside I and BTSM by A549 cells after I, 2 and 4 hours of incubation. The results were presented as the mean \pm SD $(n=6)$. ${ }^{*}<0.05$, compared with baohuoside I group. (B) Fluorescence microscope of A549 cells after 2 hours of incubation with the free fluorescent coumarin-6 and the coumarin-6-loaded mixed micelles.

Abbreviations: BTSM, baohuoside I-loaded mixed micelles consisting of TPGS and Solutol HS I5; TPGS, D- $\alpha$-tocopheryl polyethylene glycol succinate; SD, standard deviation; DAPI, 4,6-diamidino-2-phenylindole; coumarin-6-M, coumarin-6-loaded mixed micelles composed of TPGS and Solutol HS I5. 
A

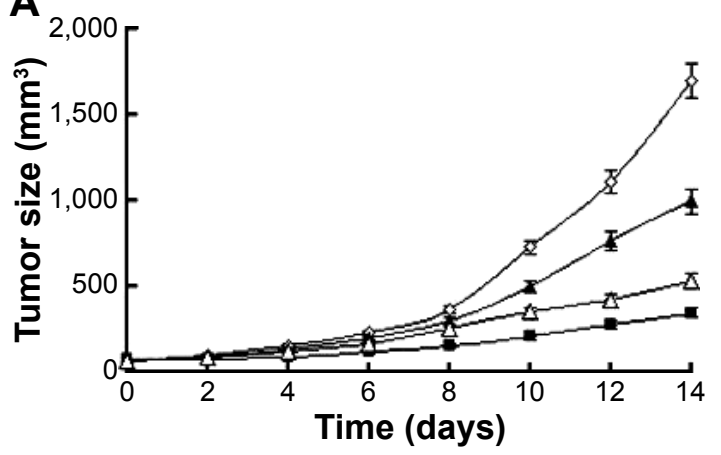

B

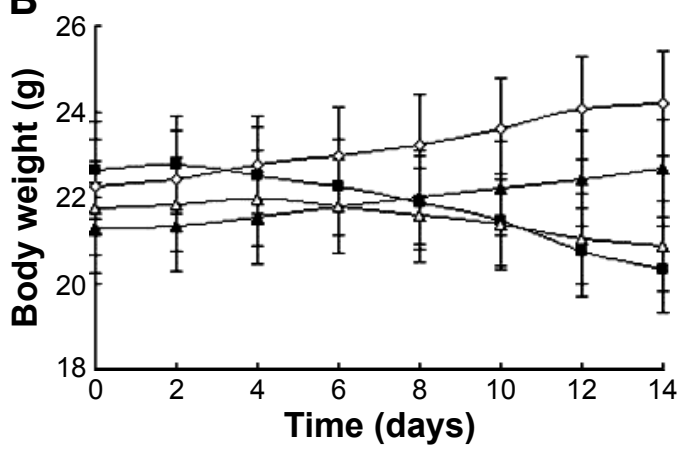

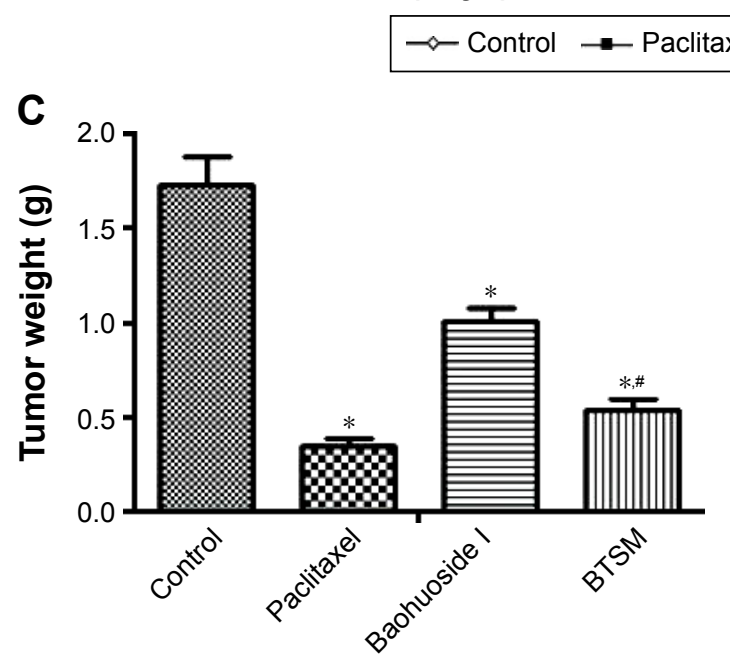

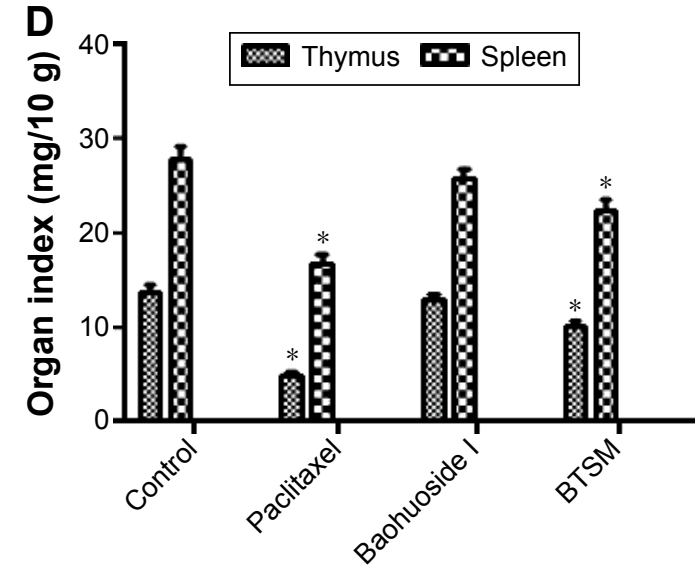

Figure 3 In vivo antitumor study of BTSM in BALB/c nude mice implanted with A549 cells.

Notes: Tumor volumes $(\mathbf{A})$ and body weight $(\mathbf{B})$ were monitored daily. Tumor weight $(\mathbf{C})$ and organ index $(\mathbf{D})$ were monitored at the end of the experiment. The results are presented as the mean $\pm S D(n=6) . * P<0.05$, compared with control group; ${ }^{*} P<0.05$, compared with baohuoside I group.

Abbreviations: BTSM, baohuoside I-loaded mixed micelles consisting of TPGS and Solutol HS I5; TPGS, D- $\alpha$-tocopheryl polyethylene glycol succinate; SD, standard deviation.

with A549 cells on the right side. Mice treated with DiR-M showed a time-dependent increase in the fluorescence signals, and they were mainly distributed on the tumor (Figure 4). Fluorescence signals were clearly observed at the tumor site and maintained for 24 hours. Therefore, with the modification of Solutol HS 15, the TPGS/Solutol HS 15 mixed micelles were efficiently delivered and retained by the lung tumors under in vivo conditions.

\section{Discussion}

The antitumor effect of flavonoids has been extensively examined. Owing to their strong anticancer effects and low toxicity, flavonoids have a great potential of being developed as anticancer drugs. ${ }^{23-25}$ Baohuoside $\mathrm{I}$ is the main active flavonoid component of Herba epimedii and shows impressive pharmacological effects. Although baohuoside I has antitumor characteristics, it has poor solubility and permeability and may be pumped out by the intestinal efflux transporters, and thus, its use for the treatment of human diseases is limited. ${ }^{26}$ To improve the solubility and cellular uptake of baohuoside I, mixed micelles were prepared. In this study, the purity of baohuoside I was $98.22 \%$, and the solubility, as determined by us, was found to be $0.0124 \mathrm{~g} / \mathrm{L}$. The solubility of baohuoside I in the micelles was $2.176 \mathrm{~g} / \mathrm{L}$, which was 175.5 times that of the baohuoside I. The structures of Solutol HS 15 and poloxamer188 show that they have the same hydrophilic chains (PEG) but different hydrophobic chains. The interaction between the two hydrophobic chains contributes to the formation of mixed micelles, which have a better stability compared to the single micelles.

The $\mathrm{IC}_{50}$ value reflected the MDR reversing ability of certain formulations. Free baohuoside I was easily effluxed from cells via the P-gp. The $\mathrm{IC}_{50}$ value of BTSM was lower than that of free baohuoside I, indicating that the mixed micelles had MDR reversing ability. TPGS and Solutol HS 15 have been used as an excipient for overcoming MDR and as an inhibitor of P-gp. Our results indicate that the superior cytotoxicity of BTSM might be attributed to a drastic increase in drug accumulation by A549 cells owing to reduced efflux and increased uptake.

The enhanced sensitization of MDR cancer to baohuoside I could be the main reason for the significant suppression of 


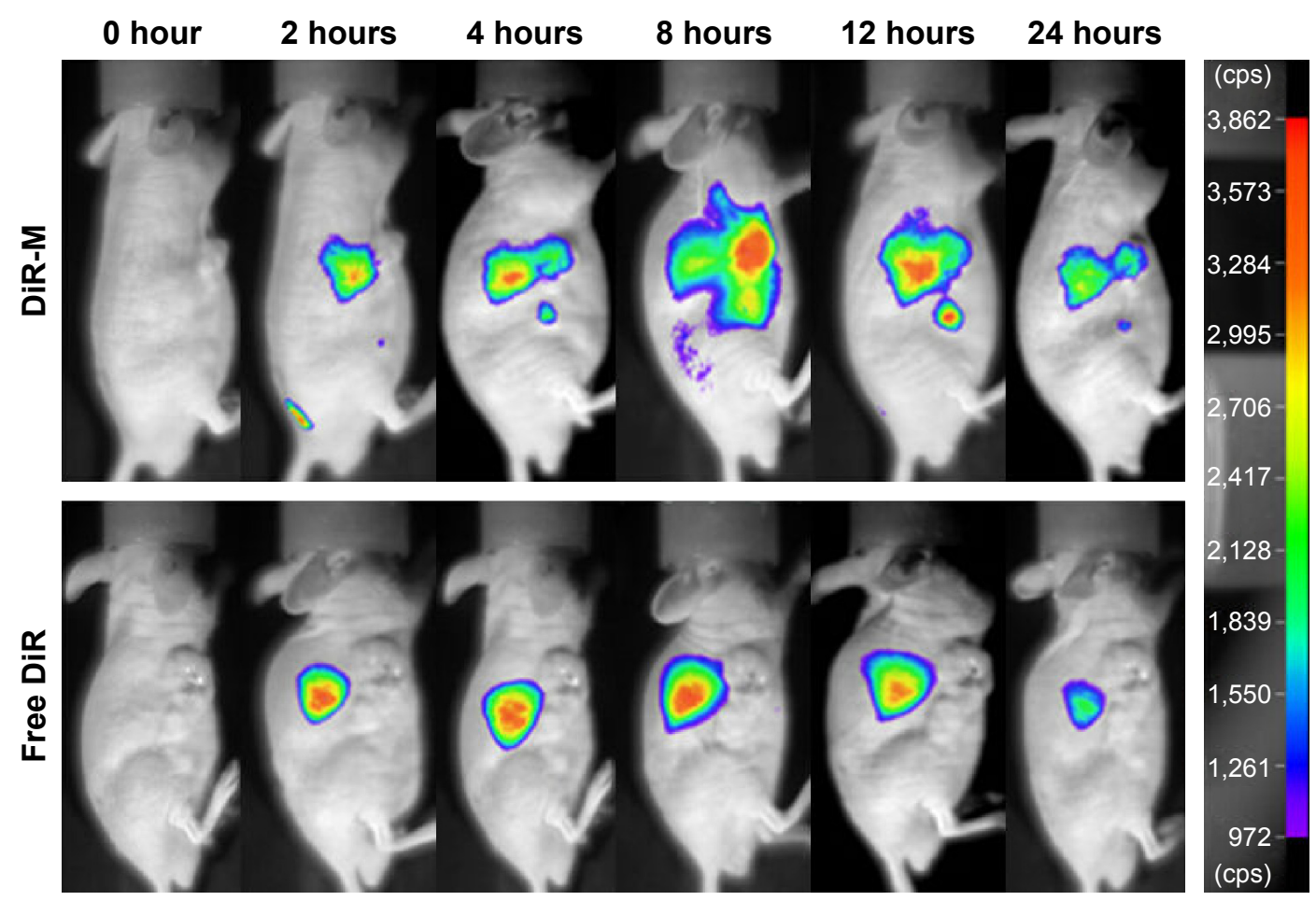

Figure 4 Fluorescence images of the mice bearing A549 cells on right side at different time points after intravenous injection of DiR-loaded mixed micelles. Abbreviations: DiR, I, I-Dioctadecyl-3,3,3,3-tetramethylindotricarbocyanine; DiR-M, DiR-loaded mixed micelles composed of TPGS and Solutol HS I5.

tumor growth in the BTSM group. Due to the suitable size and unique core-shell architecture of polymeric micelles, the micellar system could achieve a long blood circulation effect, which led to an increased local concentration of baohuoside I in the tumor tissue via the EPR effect. In addition, the change in body weight was also monitored during the experiment. The extent of weight loss induced by BTSM was smaller than that induced by paclitaxel. All these results indicate that BTSM not only led to less systemic toxicity, but also inhibited MDR tumor growth.

Coumarin-6 is a kind of lipid-soluble laser dye with high conversion efficiency and stable performance. It has been reported that the nanoparticles containing $0.05 \%$ of coumarin- 6 can be observed under the fluorescence microscope with high sensitivity. ${ }^{27}$ Therefore, in recent years, coumarin-6 is often used as a fluorescent substance, and is contained in the drug delivery system to study the cellular uptake and transport mechanism of the drug delivery system. ${ }^{28,29}$ However, the emission wavelength of coumarin-6 is too short to penetrate the tissue. Hence, it is not suitable for in vivo imaging to study the distribution in normal and target tissues. It requires a fluorescent substance with longer wavelength $(>700 \mathrm{~nm})$. DiR iodide is a fluorescent substance with an emission wavelength of $780 \mathrm{~nm}$, and the combination between $\mathrm{DiR}$ and the cell membrane is stable. ${ }^{30}$

\section{Conclusion}

In this study, we designed mixed micelles consisting of TPGS and Solutol HS 15 to entrap the poorly soluble anticancer drug baohuoside I. The baohuoside I-loaded mixed micelles (BTSM) had $85 \%$ higher EE and particle size of $\sim 60.52 \mathrm{~nm}$. Compared to free baohuoside I, baohuoside I from BTSM showed a controlled release without any initial burst release and an increased cytotoxicity toward NSCLC cells in vitro. BTSM could also enhance the cellular uptake significantly by the improvement of membrane permeability and reduction of efflux phenomenon. BTSM showed markedly higher antitumor efficacy than baohuoside I in in vivo studies. In this study, we showed that TPGS/Solutol HS 15 mixed micelles significantly increased the antitumor and targeting efficacy, and they may be a potential delivery system to maximize the use of baohuoside I as a therapeutic agent for certain pathology in humans.

\section{Acknowledgment}

This work was supported by the National Natural Science Foundation of China (81303275, 81274088).

\section{Disclosure}

The authors report no conflicts of interest in this work. 


\section{References}

1. Qian Q, Li SL, Sun E, et al. Metabolite profiles of icariin in rat plasma by ultra-fast liquid chromatography coupled to triple-quadrupole/time-offlight mass spectrometry. J Pharm Biomed Anal. 2012;66:392-398.

2. Zhai YK, Guo X, Pan YL, et al. A systematic review of the efficacy and pharmacological profile of Herba epimedii in osteoporosis therapy. Pharmazie. 2013;68:713-722.

3. Xiao HH, Fung CY, Mok SK, et al. Flavonoids from Herba epimedii selectively activate estrogen receptor alpha (ER $\alpha)$ and stimulate ERdependent osteoblastic functions in UMR-106 cells. J Steroid Biochem Mol Biol. 2014;143:141-151.

4. Song J, Shu L, Zhang Z, et al. Reactive oxygen species-mediated mitochondrial pathway is involved in baohuoside I-induced apoptosis in human non-small cell lung cancer. Chem Biol Interact. 2012; 199:9-17.

5. Kim SH, Ahn KS, Jeong SJ, et al. Janus activated kinase $2 /$ signal transducer and activator of transcription 3 pathway mediates icariside II-induced apoptosis in U266 multiple myeloma cells. Eur J Pharmacol. 2011; $1: 10-16$.

6. Jin X, Zhang ZH, Sun E, Qian Q, Tan XB, Jia XB. Preparation of a nanoscale baohuoside I-phospholipid complex and determination of its absorption: in vivo and in vitro evaluations. Int J Nanomed. 2012;7: 4907-4916.

7. Liu YQ, Yang QX, Cheng MC, Xiao HB. Synergistic inhibitory effect of icariside II with icaritin from Herba epimedii on pre-osteoclastic RAW264.7 cell growth. Phytomedicine. 2014;21:1633-1637.

8. Chen Y, Zhao YH, Jia XB, Hu M. Intestinal absorption mechanisms of prenylated flavonoids present in the heat-processed Epimedium koreanum Nakai (Yin Yanghuo). Pharm Res. 2008;25:2190-2199.

9. Jin X, Zhang ZH, Sun E, Tan XB, Zhu FX, Jia XB. A novel drugphospholipid complex loaded micelle for baohuoside I enhanced oral absorption: in vivo and in vitro evaluations. Drug Dev Ind Pharm. 2012; 39:1421-1430.

10. Jeong EJ, Liu X, Jia X, Chen J, Hu M. Coupling of conjugating enzymes and efflux transporters impact on bioavailability and drug interactions. Curr Drug Metab. 2005;6:455-468.

11. Fang J, Nakamura $\mathrm{H}$, Maeda $\mathrm{H}$. The EPR effect: unique features of tumor blood vessels for drug delivery, factors involved, and limitations and augmentation of the effect. Adv Drug Deliv Rev. 2011;63:136-151.

12. Sarika PR, James NR, Kumar PR, Raj DK, Kumary TV. Gum arabiccurcumin conjugate micelles with enhanced loading for curcumin delivery to hepatocarcinoma cells. Carbohydr Polym. 2015;134:167-174.

13. Zhao Y, Cui Y, Li Y, Li L. Stable phosphatidylcholine-bile salt mixed micelles enhance oral absorption of paclitaxel: preparation and mechanism in rats. J Drug Target. 2014;22:901-912.

14. Wang J, Mongayt D, Torchilin VP. Polymeric micelles for delivery of poorly soluble drugs: preparation and anticancer activity in vitro of paclitaxel incorporated into mixed micelles based on poly(ethylene glycol)-lipid conjugate and positively charged lipids. J Drug Target. 2005; 13:73-80.
15. Shah AR, Banerjee R. Effect of D- $\alpha$-tocopheryl polyethylene glycol 1000 succinate (TPGS) on surfactant monolayers. Colloids Surf B Biointerfaces. 2011;85:116-124.

16. Zhang ZP, Tan SW, Feng SS. Vitamin E TPGS as a molecular biomaterial for drug delivery. Biomaterials. 2012;33:4889-4906.

17. Wang AT, Liang DS, Liu YJ, Qi XR. Roles of ligand and TPGS of micelles in regulating internalization, penetration and accumulation against sensitive or resistant tumor and therapy for multidrug resistant tumors. Biomaterials. 2015;53:160-172.

18. Rajebahadur M, Zia H, Nues A, et al. Mechanistic study of solubility enhancement of nifedipine using vitamin E TPGS or solutol HS-15. Drug Deliv. 2006;13:201-206.

19. Coon JS, Knudson W, Clodfelter K, et al. Solutol HS 15, nontoxic polyoxyethylene esters of 12-hydro-xystearic acid, reverses multidrug resistance. Cancer Res. 1991;51:897-902.

20. Singh MS, Lamprecht A. P-glycoprotein inhibition of drug resistant cell lines by nanoparticles. Drug Dev Ind Pharm. 2015;11:1-7.

21. Engel A, Oswald S, Siegmund W, Keiser M. Pharmaceutical excipients influence the function of human uptake transporting proteins. Mol Pharm. 2012;9:2577-2581.

22. Kim TH, Mount CW, Dulken BW, et al. Filamentous, mixed micelles of triblock copolymers enhance tumor localization of indocyanine green in a murine xenograft model. Mol Pharm. 2012;9:135-143.

23. Martinez-Perez C, Ward C, Cook G, et al. Novel flavonoids as anti-cancer agents: mechanisms of action and promise for their potential application in breast cancer. Biochem Soc Trans. 2014;42:1017-1023.

24. Ravishankar D, Rajora AK, Greco F, Osborn HM. Flavonoids as prospective compounds for anticancer therapy. Int J Biochem Cell Biol. 2013; 45:2821-2831.

25. Orlikova B, Menezes JC, Ji S, Kamat SP, Cavaleiro JA, Diederich M. Methylenedioxy flavonoids: assessment of cytotoxic and anti-cancer potential in human leukemia cells. Eur J Med Chem. 2014;84:173-180.

26. Chen Y, Jia X, Tan X, Fan C, Hu M. Absorption and metabolism of flavonoids in Herba epimedii via rat intestinal perfusion model. Zhongguo Zhong Yao Za Zhi. 2009;34:2928-2931.

27. Panyama J, Sahoo SK, Prabha S, et al. Fluorescence and electron microscopy probes for cellular and tissue uptake of poly (D, L-lactideco-glycolide) nanoparticles. Int J Pharm. 2003;262:1-11.

28. Makhlof A, Tozuka Y, Takeuchi H. pH-sensitive nanospheres for colon-specific drug delivery in experimentally induced colitis rat model. Eur J Pharm Biopharm. 2009;72:1-8.

29. Hu KL, Li JW, Shen YH, et al. Lactoferrin-conjugated PEG-PLA nanoparticles with improved brain delivery: In vitro and in vivo evaluations. $J$ Control Release. 2009;134:55-61.

30. Wen Z, Yan Z, He R, et al. Brain targeting and toxicity study of odorranalectin-conjugated nanoparticles following intranasal administration. Drug Deliv. 2011;18:555-561.
International Journal of Nanomedicine

\section{Publish your work in this journal}

The International Journal of Nanomedicine is an international, peerreviewed journal focusing on the application of nanotechnology in diagnostics, therapeutics, and drug delivery systems throughout the biomedical field. This journal is indexed on PubMed Central,

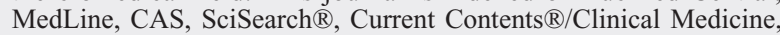

\section{Dovepress}

Journal Citation Reports/Science Edition, EMBase, Scopus and the Elsevier Bibliographic databases. The manuscript management system is completely online and includes a very quick and fair peer-review system, which is all easy to use. Visit http://www.dovepress.com/ testimonials.php to read real quotes from published authors. 